\title{
HYDROGEN-POOR CIRCUMSTELLAR SHELLS FROM PULSATIONAL PAIR-INSTABILITY SUPERNOVAE WITH RAPIDLY ROTATING PROGENITORS
}

\author{
E. Chatzopoulos and J. Craig Wheeler \\ Department of Astronomy, University of Texas at Austin, Austin, TX, USA; manolis@astro.as.utexas.edu \\ Received 2012 June 4; accepted 2012 October 17; published 2012 November 16
}

\begin{abstract}
In certain mass ranges, massive stars can undergo a violent pulsation triggered by the electron/positron pair instability that ejects matter, but does not totally disrupt the star. After one or more of these pulsations, such stars are expected to undergo core-collapse to trigger a supernova ( $\mathrm{SN}$ ) explosion. The mass range susceptible to this pulsational phenomena may be as low as 50-70 $M_{\odot}$ if the progenitor is of very low metallicity and rotating sufficiently rapidly to undergo nearly homogeneous evolution. The mass, dynamics, and composition of the matter ejected in the pulsation are important aspects for determining the subsequent observational characteristics of the explosion. We examine the dynamics of a sample of stellar models and rotation rates and discuss the implications for the first stars, for LBV-like phenomena, and for superluminous SNe. We find that the shells ejected by pulsational pair-instability events with rapidly rotating progenitors ( $>30 \%$ the critical value) are hydrogen-poor and heliumand oxygen-rich.
\end{abstract}

Key words: stars: evolution - stars: mass-loss - stars: rotation - supernovae: general

\section{INTRODUCTION}

Very massive stars will be subject to dynamical instability triggered by the formation of electron positron pairs (Rakavy \& Shaviv 1968; Barkat et al. 1967; Fraley 1968). In some circumstances, this instability will lead to violent contraction of the oxygen core, ignition of the oxygen, and total disruption of the star as a pair-instability supernova (PISN). At somewhat more modest mass, the collapse and burning will lead to the ejection of a shell of matter, but not total disruption: a pulsational pair instability supernova (PPISN; Barkat et al. 1967; Heger \& Woosley 2002; Woosley et al. 2007, hereafter WBH07). In the case of zero rotation, WBH07 determined that stars with zero-age main-sequence (ZAMS) masses in the range $95-130 M_{\odot}$ become PPISN. Chatzopoulos \& Wheeler (2012; hereafter CW12) explored the boundary between core collapse, PPISN, and PISN for the case of zero metallicity, as a function of the rate of rotation of the progenitor (see also Yoon et al. 2012). CW12 checked the dynamics of their rotating stellar evolution models by computing one-dimensional (1D), non-rotating hydrodynamic models to confirm that they underwent core collapse, PPISN, or PISN. These models are not completely self-consistent since they ignore the dynamical effects of rotation, but are reasonably self-consistent in the sense that they map the structure of nominally rotating but "shellular" stellar models into spherically symmetric dynamic models.

The masses of the PPISN progenitors are sensitive to the effect of mass loss. Mass loss is a rather uncertain process in the case of very massive stars and can happen continuously in the form of radiatively driven winds or gravity waves (Quataert \& Shiode 2012), episodically via shell ejections and mechanically due to rapid rotation. Furthermore, mass loss is a strong function of metallicity and higher metallicities will prevent initially massive stars from encountering pair instability in the core (Yoon et al. 2006; Langer et al. 2007). Langer et al. (2007) estimate that rapidly rotating PISN progenitors may be possible for metallicities $Z<10^{-5} Z_{\odot}$ and less likely in the local universe. Despite the small expected rate of PISN and PPISN events in the local universe, the possibility of those events taking place in metal-poor environments is non-zero and potential candidates have been discussed (SLSN 2007bi; Gal-Yam et al. 2009). In addition, we note that chemical mixing induced by rapid rotation is not the only way to make hydrogen-poor PPISN progenitors. Hydrogen envelope stripping via stellar winds from very massive stars is another possibility that has been discussed, although it might also require low metallicity (Langer et al. 2007; Yoshida \& Umeda 2011). The results presented here may be more relevant to early universe population III PPISN progenitors, but may be used as a guideline for potentially similar local universe, low-metallicity events (Neill et al. 2011).

The dynamics can give insight into the expected behavior of the resulting configuration that may have direct implications for observations of the first stars by the James Webb Space Telescope. In addition, the dynamical ejection of shells may be related to the observed impulsive mass ejection associated with luminous blue variables (Smith \& Owocki 2006; Smith et al. 2007). The PPISN phenomenon may also be relevant to various manifestations of superluminous supernovae (SLSNe; see Gal-Yam 2012 for a review). Some of these events display the characteristics of Type IIn supernovae and are clearly the result of the collision of an underlying explosion with a dense, optically thick circumstellar medium (CSM; Chevalier 1982; Chevalier \& Fransson 1994; Ofek et al. 2010; Moriya et al. 2011; Chevalier \& Irwin 2011; Chatzopoulos et al. 2012). Other SLSNe show little or no hydrogen (Quimby et al. 2011) and little sign of circumstellar interaction. An outstanding issue is whether a rapidly expanding hydrogen-deficient CSM would suppress the narrow lines normally thought to accompany CSM interaction (Blinnikov \& Sorokina 2010; R. M. Quimby 2012, private communication). Yet other SLSNe show no hydrogen or helium, evidence for nickel and cobalt and a light curve (LC) that could be powered by radioactive decay and hence might be candidates for full-fledged PISN. An example is SLSN 2007bi (Gal-Yam et al. 2009). While the ejecta mass for SLSN 2007bi seems adequate to conform to predictions for PISN, the ejecta mass of the otherwise similar SLSN 2010kd (J. Vinko et al. 2012 , in preparation) seems too low to satisfy this criterion. If SLSN 2010kd cannot be a PISN, then some question arises as to whether or not there are alternative explanations for SLSN 2007bi, for instance the collision of a supernova (SN) 
with a hydrogen- and a helium-deficient CSM that, as above, might be expanding sufficiently rapidly to broaden and mute narrow emission lines. SLSN 2006oz shows evidence for such a hydrogen-deficient CSM (Leloudas et al. 2012).

There is thus considerable interest in understanding the mass, dynamics, and composition of the matter that might be ejected in PPISN events. In this paper, we present the details of the dynamics of some PPISN events computed by CW12. Section 2 describes our assumptions and models, Section 3 gives the results. Finally, Section 4 discusses our conclusions.

\section{MODELS}

To study the dynamics of PPISN events, we select some of the zero metallicity models studied by CW12 plus a $110 M_{\odot}$ with $Z=10^{-3} Z_{\odot}$. We concentrate on the CW12 models with masses of 60,75 , and $80 M_{\odot}$ with ZAMS rotation of $50 \%, 50 \%$, and $30 \%$ of the critical value, $\Omega_{\text {crit }}$, respectively, where $\Omega_{\text {crit }}=$ $(g(1-\Gamma) / R)^{1 / 2}$ and $g=G M / R^{2}$ is the gravitational acceleration at the "surface" of the star, $G$ is the gravitational constant, $M$ is the mass, $R$ is the radius of the star, and $\Gamma=L / L_{\mathrm{Ed}}$ is the Eddington factor where $L$ and $L_{\mathrm{Ed}}$ are the total radiated luminosity and the Eddington luminosity, respectively. All of the models were evolved from the ZAMS up to the time of maximum compression with radiatively and mechanically driven mass loss included, right before the core density and temperature enter the $\Gamma<4 / 3$ dynamically unstable regime, with the Modules for Experiments in Stellar Astrophysics stellar evolution code (MESA version 4298; Paxton et al. 2011). MESA accounts for the effects of angular momentum transport and chemical mixing due to rotation and magnetic fields as parameterized by Heger et al. (2005) based on the prescriptions of Spruit (1999, 2002). For more details on the physics employed in the MESA models used here, see CW12. Although CW12 considered both models without mass loss and models with mass loss included, we note that the neglect of the effects of mass loss in the evolution of some PPISN and PISN progenitor models will lead to super-critical rotation and improper treatment of angular momentum transport that would affect our results on the composition and properties of the ejected PPISN shells. All zero metallicity models presented here were considered in CW12 to estimate the effect of mass loss on the minimum ZAMS mass of rotating PISN and PPISN progenitors (dashed lines in their Figure 5). In addition to those models, we also considered the evolution of a $110 M_{\odot}$ star rotating at $30 \%$ of the critical value with metallicity $1 / 1000$ that of the Sun in order to investigate the characteristics of PPISN in low, but non-zero, metallicity environments, which could be relevant to some SLSNe observed in metal-poor galaxies. For radiatively driven mass loss we used the prescriptions of Glebbeek et al. (2009) and de Jager et al. (1988). Rotationally induced mass loss is equal to $\dot{M}_{\text {rot }}=\dot{M}_{\text {no-rot }} /\left(1-\Omega / \Omega_{\text {crit }}\right)^{0.43}$ where $\dot{M}_{\text {no-rot }}$ is the mass loss rate in the case of zero rotation, due to the effect of radiatively driven winds (Heger et al. 2000). The characteristics of all evolved MESA progenitor models such as the final (pre-PPISN) rotation rate $\left(\Omega / \Omega_{\text {crit }, f}\right)$, radius $\left(R_{f}\right)$, carbonoxygen core gravitational binding energy $\left(-E_{B, f}\right)$, and carbon-oxygen core mass $\left(M_{\mathrm{CO}, f}\right)$ are summarized in Table 1.

The nearly hydrostatic MESA models were then mapped into the multi-dimensional, adaptive mesh refinement hydrodynamics code FLASH (Fryxell et al. 2000) in order to perform 1D simulations to follow the dynamical collapse and subsequent pulse and ejection of material as well as nucleosynthesis. The transition from MESA to FLASH is an operationally smooth one because the two codes employ the same equation of state
Table 1

Physical Characteristics of the Pre-PPISN Models Used in This Work

\begin{tabular}{lcccccc}
\hline \hline $\begin{array}{l}M_{\text {ZAMS }} \\
\left(M_{\odot}\right)\end{array}$ & $\begin{array}{c}M_{f} \\
\left(M_{\odot}\right)\end{array}$ & $\Omega / \Omega_{\text {crit,ZAMS }}$ & $\Omega / \Omega_{\text {crit }, f}$ & $\begin{array}{c}R_{f} \\
\left(10^{11} \mathrm{~cm}\right)\end{array}$ & $\begin{array}{c}-E_{B, f} \\
\left(10^{52} \mathrm{erg}\right)\end{array}$ & $\begin{array}{c}M_{\mathrm{CO}, f} \\
\left(M_{\odot}\right)\end{array}$ \\
\hline 60 & 46 & 0.50 & 1.00 & 1.10 & 0.43 & 41 \\
70 & 47 & 0.50 & 1.00 & 0.41 & 0.73 & 46 \\
80 & 58 & 0.30 & 1.00 & 0.49 & 1.16 & 55 \\
$110^{\mathrm{a}}$ & 41 & 0.30 & 1.00 & 0.58 & 0.40 & 41 \\
\hline
\end{tabular}

Notes. Quantities with the subscript " $f$ " denote pre-PPISN values.

${ }^{\text {a }}$ For this model the initial metallicity was $Z=10^{-3} Z_{\odot}$.

(HELM; Timmes \& Swesty 2000) and the same nuclear reaction network. In addition, appropriate mesh refinement selections at initialization were made in FLASH in order to achieve the desired resolution for accurate calculation of the core compression and subsequent shock formation and core oxygen burning. The simulation box size for all FLASH simulations was chosen to be $\sim 10$ times larger than the stellar radius of the relevant model in order to sufficiently follow the ejected shell and determine the mass of the unbound material after the pulse is complete.

We limited our study to ZAMS rotation rates $\leqslant 50 \% \Omega_{\text {crit }}$ because the effects of higher rotation in the hydrodynamic equilibrium of the models mapped to $F L A S H$ become especially important. The equation for hydrostatic equilibrium for rotating stars (Lebovitz 1967; Maeder \& Meynet 2011, and references therein) can be expressed as

$$
\frac{1}{\rho} \vec{\nabla} P=-\vec{\nabla} \Phi+\frac{1}{2} \Omega^{2} \vec{\nabla}(r \sin \theta)^{2},
$$

where $\rho$ is the local density, $P$ is the local pressure, $\Phi$ is the gravitational potential, $\Omega$ is the local angular velocity, $r$ is the distance from the center of the star, and $\theta$ is the colatitude (angular distance from the pole of the star). Equation (1) can be rewritten as follows in the case of one dimension and across the equator $(\theta=\pi / 2)$ and by changing variable from $d r$ to fluid element mass $d m_{r}=4 \pi r^{2} d r$ :

$$
\frac{d P}{d m_{r}}=-\frac{G m_{r}}{4 \pi r^{4}}+\frac{\Omega^{2}}{4 \pi r},
$$

where we have used $-\vec{\nabla} \Phi=\left(G m_{r} / r^{2}\right) \vec{r} / r$. Now we can consider the following ratio in order to assess the effects of rotation in hydrostatic equilibrium:

$$
\ell=\frac{\frac{\Omega^{2}}{4 \pi r}}{\left|\frac{d P}{d m_{r}}+\frac{G m_{r}}{4 \pi r^{4}}\right|} .
$$

For zero rotation $(\Omega=0), \ell=0$. A case of $\ell$ close to unity would imply that the effects of rotation are comparable to the combined effects of gravity and internal pressure; therefore rotation should not be ignored in the hydrodynamic calculations. In general, the larger the value of $\ell$ the more important the effects of rotation to hydrostatic equilibrium become. For the MESA models mapped to FLASH in the cases of ZAMS rotation of $30 \%$ and even $50 \%$ of the critical rotation, $\ell$ in the core was limited to less than 0.05 , with $\ell=0.05$ the peak value for ZAMS $\Omega / \Omega_{\text {crit }}=0.5$ and $\ell=2 \times 10^{-4}$ representative for ZAMS $\Omega / \Omega_{\text {crit }}=0.3$. In more extreme cases of rotation (ZAMS $\Omega / \Omega_{\text {crit }}=0.8$, also presented in $\mathrm{CW} 12), \ell$ becomes close to unity and the effects of rotation cannot be ignored. Models with this very high rate of rotation collapse in a dynamical timescale when mapped into FLASH. 


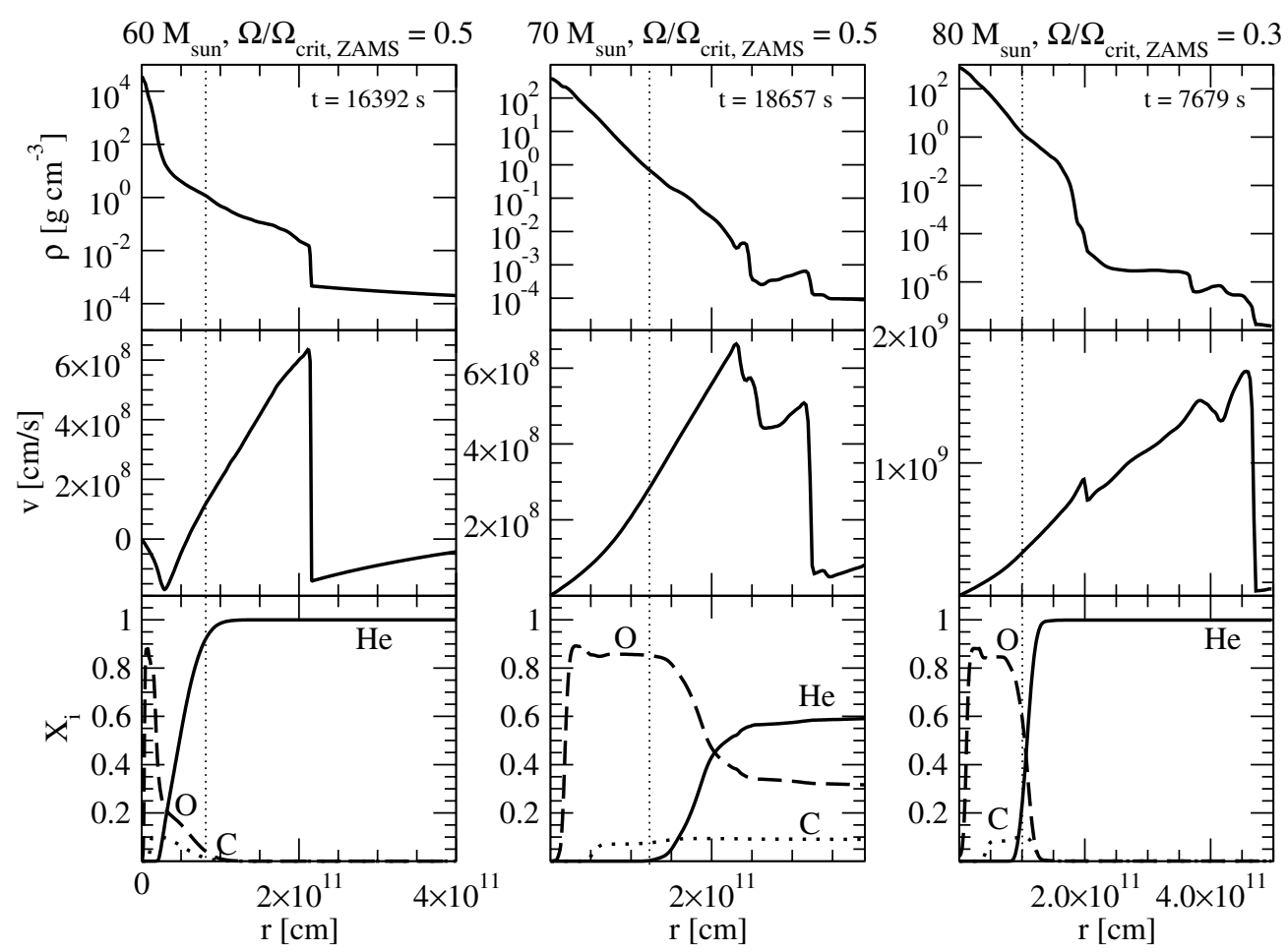

Figure 1. Density (upper panels), velocity (middle panels, horizontal), and composition profiles (lower panels) for shells ejected by PPISNe of progenitor masses $60 M_{\odot}$ (left panels), $70 M_{\odot}$ (middle panels, vertical), and $80 M_{\odot}$ (right panels) with ZAMS rotational velocities 50\%, 50\%, and 30\% the critical value accordingly. In the composition profiles, the solid curves show the mass fraction of helium, the dashed curves the mass fraction of oxygen, and the dotted curves the mass fraction of carbon. The time since the first PPISNe pulse in each case is given in the upper panels. In all panels, the dashed vertical lines indicate the radii above which the material is gravitationally unbound.

The models with ZAMS rotation of $30 \%$ and $50 \%$ of the critical value that were mapped to FLASH within the scope of this project remain stable over long timescales (greater than their corresponding free-fall dynamical collapse timescales) before a significant fraction of their cores encounters the pair-formation regime of $\Gamma<4 / 3$ and collapse leading to PPISN shell ejection.

In this project, we study only the first shell ejections due to PPISNe. As WBH07 discussed, subsequent pulses may be encountered by a massive star depending on its initial carbon/oxygen core mass. Multiple shell ejections will interact with each other and ultimately the ejecta of the final SN explosion will interact with them, too, resulting in several luminous transient events over the duration of decades up to centuries before stellar death. Since we are just performing 1D hydrodynamic simulations, we ignore the effect of rotation on the shape of the ejected shell.

\section{RESULTS}

We post-processed the FLASH simulation files for the three models from CW12 discussed above as well as the $110 M_{\odot}$, $Z=10^{-3} Z_{\odot}$ model in order to get measures of the mass lost due to the violent PPISN as well as to determine the physical characteristics of the shells ejected as a function of increasing ZAMS mass and rotational velocity as well as metallicity. Figure 1 presents the distributions of density, velocity, and chemical composition for all models. Details of the characteristics of the shells ejected by the first pulse in each case are given in Table 2 where the shell mass $\left(M_{\mathrm{sh}}\right)$, shell kinetic energy $\left(E_{K, \mathrm{sh}}\right)$, typical shell velocity $\left(v_{\mathrm{sh}}=\left(2 E_{K, \mathrm{sh}} / M_{\mathrm{sh}}\right)^{1 / 2}\right)$, and the total masses of helium $\left(M_{\mathrm{He}, \mathrm{sh}}\right)$, carbon $\left(M_{\mathrm{C}, \mathrm{sh}}\right)$, and oxygen $\left(M_{\mathrm{O}, \mathrm{sh}}\right)$ within the ejected shells are presented. The
Table 2

Physical Characteristics of the Shells Ejected by the PPISN Models Discussed in This Work

\begin{tabular}{lccccccc}
\hline \hline $\begin{array}{l}M_{\text {ZAMS }} \\
\left(M_{\odot}\right)\end{array}$ & $\Omega / \Omega_{\text {crit ZAMS }}$ & $\begin{array}{c}M_{\text {sh }} \\
\left(M_{\odot}\right)\end{array}$ & $\begin{array}{c}E_{K, \text { sh }} \\
\left(10^{51} \mathrm{erg}\right)\end{array}$ & $\begin{array}{c}v_{\text {sh }}{ }^{\mathrm{a}} \\
\left(\mathrm{km} \mathrm{s}^{-1}\right)\end{array}$ & $\begin{array}{c}M_{\mathrm{He}, \mathrm{sh}} \\
\left(M_{\odot}\right)\end{array}$ & $\begin{array}{c}M_{\mathrm{C}, \mathrm{sh}} \\
\left(M_{\odot}\right)\end{array}$ & $\begin{array}{c}M_{\mathrm{O}, \mathrm{sh}} \\
\left(M_{\odot}\right)\end{array}$ \\
\hline 60 & 0.5 & 1.9 & 0.25 & 3636.48 & 1.71 & 0.06 & 0.13 \\
70 & 0.5 & 3.9 & 0.31 & 2826.42 & 0.23 & 0.81 & 2.86 \\
80 & 0.3 & 7.3 & 0.48 & 2570.68 & 0.32 & 0.62 & 6.36 \\
$110^{\mathrm{b}}$ & 0.3 & 3.1 & 0.09 & 1607.59 & 0.17 & 0.78 & 2.15 \\
\hline
\end{tabular}

Notes.

a The average shell velocity is obtained by making use of the formula $v_{\mathrm{sh}}=$ $\sqrt{\left(2 E_{K, \mathrm{sh}} / M_{\mathrm{sh}}\right)}$.

${ }^{\mathrm{b}}$ For this model the initial metallicity was $Z=10^{-3} Z_{\odot}$.

masses and kinetic energies of the shells were calculated by determining how much mass is gravitationally unbound after the pulse was complete. We considered the matter to be gravitationally unbound above radii for which $E_{K}+E_{\mathrm{int}}-U_{G}>$ 0 , where $E_{K}$ is the kinetic, $E_{\text {int }}$ is the internal, and $U_{G}$ is the gravitational binding energy of the simulated material.

We see from Table 2 that for fixed initial ZAMS rotational velocity and increasing mass, the ejected PPISN shells are more massive and have higher kinetic energies. On the other hand, increasing rotation leads to the ejection of shells of lower mass: $\sim 7 M_{\odot}$ in the case of ZAMS $\Omega / \Omega_{\text {crit }}=0.3$ (for the $80 M_{\odot}$ model) and $2-4 M_{\odot}$ in the case of ZAMS $\Omega / \Omega_{\text {crit }}=0.5$ (for the 60 and $70 M_{\odot}$ models). WBH07 calculated an ejected shell of $17.6 M_{\odot}$ in the case of a non-rotating $60 M_{\odot}$ oxygen core. This shell mass is larger than that of our $30 \%$ critically rotating $80 M_{\odot}$ model (which forms a $55 M_{\odot}$ oxygen core mass) and 

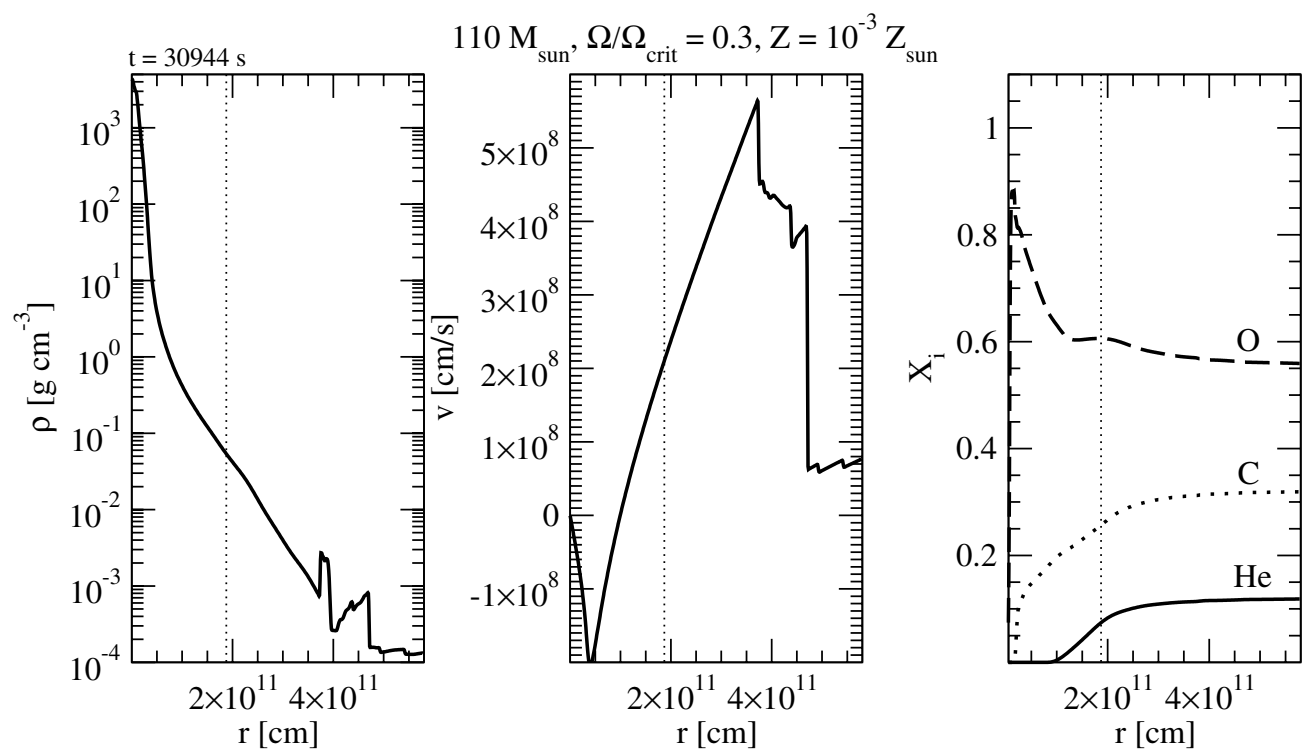

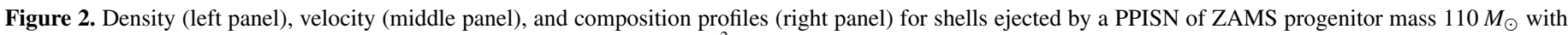

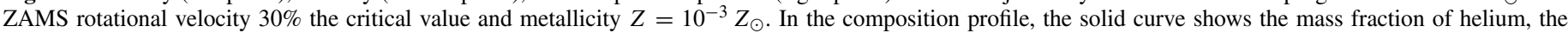

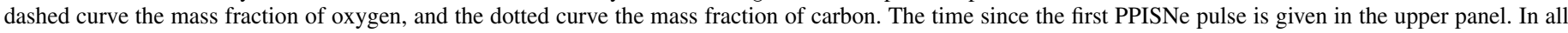
panels, the dashed vertical lines indicate the radii above which the material is gravitationally unbound.

much larger than that of our $50 \%$ critically rotating $70 M_{\odot}$ model (which forms a $56 M_{\odot}$ oxygen core).

Pre-SN mass loss lead to almost entirely stripped carbonoxygen cores for the zero metallicity CW12 models with $70 M_{\odot}$, $\Omega / \Omega_{\text {crit }}=0.5$ and $80 M_{\odot}, \Omega / \Omega_{\text {crit }}=0.3$. Mass loss results in differences in the overall rotationally induced mixing efficiency, which is affected by angular momentum loss, and differences in the final structure and composition of the progenitor star and the PPISN shell. The typical mass of helium within the ejected PPISN shell ranges between 0.3 and $1.3 M_{\odot}$ (Table 2 ), a value that is in good agreement with the results presented in Table 5 of Yoon et al. (2012). In accordance, the oxygen and carbon abundances in the PPISN shells are generally enhanced since the shell now probes deeper layers in the star that extend to the carbon-oxygen core.

In Figure 1 (lower panels, horizontally), we illustrate the composition of the ejected shells. In the case of moderate rotation (ZAMS $\Omega / \Omega_{\text {crit }}=0.3$ ) the outer regions of the progenitor stars are helium-rich, with traces of oxygen and carbon present in deeper layers. As a result, the composition of the ejected PPISN shells is predominantly He with small traces of oxygen present in their inner parts. In the case of the $70 M_{\odot}$, $\Omega / \Omega_{\text {crit }}=0.5$ model, the PPISN shell, though still helium-rich, is significantly enriched mainly with oxygen but also with some carbon. In some cases, the oxygen mass fractions can be up to 0.5 or more. In all rotating cases, the shells are hydrogen-poor. The outer layers of the stars after their first PPISN are even more enhanced in oxygen and carbon; therefore, subsequent shell ejections are expected to be even more oxygen-rich, potentially leading to shell collisions of oxygen-rich material. The luminous output from this kind of CSM interaction is not necessarily going to be similar to that observed in cases of hydrogenrich CSM interaction. Emission lines of hydrogen and, in some cases, of helium will be absent in the spectrum of oxygen-rich events.

The $110 M_{\odot}$ model, with $Z=10^{-3} Z_{\odot}$ and ZAMS rotation $30 \%$ the critical value, lost the larger fraction of its initial mass to strong radiatively driven winds combined with rotationally induced mass loss, which left it with a completely stripped $\sim 41 M_{\odot} \mathrm{C} / \mathrm{O}$ core, right within the range of PPISN. The PPISN pulse was followed hydrodynamically in FLASH and the relevant dynamics are detailed in Table 2 and in Figure 2 where the density, velocity, and chemical composition of the unbound PPISN shell are shown at time $t \simeq 31,000 \mathrm{~s}$ after the pulse. In reality, the ejected PPISN shell from this model would collide with the previously expelled $69 M_{\odot}$ hydrogen/helium shell from the progenitor star, leading to a potentially longlasting SN ejecta-CSM interaction and an associated long LC duration. The effect of progenitor metallicity in the final ejected PPISN can be seen by comparing the zero metallicity $60 M_{\odot}$, $\Omega / \Omega_{\text {crit }}=0.5$ model with the $Z=10^{-3} Z_{\odot}, 110 M_{\odot}, \Omega / \Omega_{\text {crit }}=$ 0.3 model since both models make $\mathrm{C} / \mathrm{O}$ cores of the same mass $\left(41 M_{\odot}\right)$. We find that the PPISN shell of the $Z=10^{-3} Z_{\odot}$ model is more significantly enhanced in carbon and oxygen and more depleted in helium than the zero metallicity model mainly due to the fact that deeper layers are probed as a result of extreme mass loss for the $10^{-3} Z_{\odot}$ model. In addition, we find a larger PPISN shell with a smaller kinetic energy associated with the $10^{-3} Z_{\odot}$ model. The fact that the PPISN phenomenon is possible for non-zero metallicities that may be relevant to metal-poor dwarf galaxies means that these brilliant events may be related to some nearby, hydrogen-poor SLSNe such as SN 2007bi.

\section{DISCUSSION AND CONCLUSIONS}

In this paper, we have discussed the properties of shells ejected by massive $\left(60-80 M_{\odot}\right)$, rotating $(30 \%-50 \%$ the critical value on the ZAMS), stars with zero (and one case of $10^{-3} Z_{\odot}$ ) metallicity encountering PPISNe for the first time. We find that for increasing PPISN progenitor rotational velocities the resulting pulses are less energetic and shells of smaller masses but rich in helium, carbon, and oxygen are ejected. For the range of models considered here, the masses of the ejected shells vary from $\sim 2 M_{\odot}$ for higher rotation values all the way up to $\sim 7 M_{\odot}$ for lower rotation. We find that the shells from the 
first PPISN ejections are all rich in helium, oxygen, and carbon in contrast to hydrogen-rich shells ejected in non-rotating cases (WBH07). We note, however, that subsequent pulses in the case of zero rotation might also lead to helium-rich shells, since deeper layers of the star are probed. Zero-rotation models are not expected to lead to shells with significantly enhanced carbon and oxygen as is the case for rotating progenitors. The ejection of hydrogen-poor shells from massive population III stars in the early universe might have important implications for the composition of the interstellar medium in these epochs.

Our results imply that rotationally induced chemical mixing (mainly due to meridional circulation and the Spruit-Tayler mechanism for the effects of magnetic fields) in zero-metallicity massive stars leads to homogeneous evolution and larger carbon/oxygen core masses before encountering pair instability than do non-rotating models of the same mass, as shown in CW12 (see also Yoon et al. 2012). We also examined the case of a low-metallicity $\left(Z=10^{-3} Z_{\odot}\right), 110 M_{\odot}$ star which produces an entirely stripped $\left(41 M_{\odot}\right) \mathrm{C} / \mathrm{O}$ core and encounters PPISN which leads to the ejection of a $\sim 3 M_{\odot}$ shell that is significantly enhanced in carbon and oxygen. This model was run to indicate that the PPISN phenomenon leading to hydrogen-poor ejected shells might also be relevant to low-metallicity environments such as dwarf galaxies that seem to be the host environments for some hydrogen-poor SLSNe. The strong chemical mixing initially stirs helium and later oxygen and carbon to the outer layers while dredging hydrogen inward to the core. When the carbon/oxygen cores of those stars encounter PPISN they eject those helium and metal-enriched outer layers therefore chemically enriching the surrounding CSM. Subsequent pulses may be even richer in carbon and oxygen since they probe the inner regions of the star, leading to collisions of hydrogen-poor shells. Ultimately, the final SN explosion takes placed embedded within this chemically enriched CSM and the SN ejecta interact with it.

This kind of hydrogen-poor CSM interaction is not necessarily going to possess the same observational characteristics as hydrogen-rich CSM interaction. Hydrogen-rich CSM interaction seems to be related to Type IIn SNe, the spectra of which show narrow emission lines of hydrogen and, sometimes, weaker emission lines of helium. SLSN events such as SLSN 2006tf (Smith et al. 2008), SLSN 2006gy (Smith et al. 2007, 2010), and SLSN 2008es (Gezari et al. 2009; Miller et al. 2009) seem to fall into this category. On the other hand, recent discoveries of SLSNe with no signs of hydrogen in their spectrum (Quimby et al. 2011; Leloudas et al. 2012) might indicate that not all CSM interaction involves hydrogen-rich material. Additionally, some of those hydrogen-poor events show an early precursor plateau in their LCs (Blinnikov \& Sorokina 2010; Dessart et al. 2011; Leloudas et al. 2012).

In this context, a hydrogen-poor CSM interaction might also be an alternative explanation for the nature of SLSN 2007bi (Gal-Yam et al. 2009), which is considered the strongest observed candidate for PISN. At first, the CSM interaction model for this event was ruled out due its spectral characteristics showing no typical signs of hydrogen-rich interaction, given the absence of narrow hydrogen lines from any of the spectra obtained. The optical spectrum predicted for helium/carbon/oxygen-rich CSM interaction, which could result from PPISNe with rapidly rotating progenitors, is unexplored, but it must, perforce, be free of hydrogen features. For this reason, E. Chatzopoulos et al. (2012, in preparation) will present a semi-analytical CSM interaction model fit to the observed LC of SLSN 2007bi, considering this to be a possible alternative model. Future multi-group radiation hydrodynamics simulations of such events are expected to shed more light on the issue.

We thank the anonymous referee for valuable comments and suggestions that helped significantly improve this paper. We thank the MESA team for making this valuable tool readily available and especially thank Bill Paxton for his ready advice and council in running the code. We thank Volker Bromm and Milos Milosavljevic for discussions on the topic and Sean Couch and Christopher Lindner for offering advice on FLASH dynamics. This research is supported in part by NSF AST-1109801. E.C. wishes to thank the University of Texas Graduate School for the William C. Powers fellowship given in support of his studies.

\section{REFERENCES}

Barkat, Z., Rakavy, G., \& Sack, N. 1967, Phys. Rev. Lett., 18, 379 Blinnikov, S. I., \& Sorokina, E. I. 2010, arXiv:1009.4353

Chatzopoulos, E., \& Wheeler, J. C. 2012, ApJ, 748, 42

Chatzopoulos, E., Wheeler, J. C., \& Vinko, J. 2012, ApJ, 746, 121

Chevalier, R. A. 1982, ApJ, 258, 790

Chevalier, R. A., \& Fransson, C. 1994, ApJ, 420, 268

Chevalier, R. A., \& Irwin, C. M. 2011, ApJ, 729, L6

de Jager, C., Nieuwenhuijzen, H., \& van der Hucht, K. A. 1988, A\&AS, 72, 259

Dessart, L., Hillier, D. J., Livne, E., et al. 2011, MNRAS, 414, 2985

Fraley, G. S. 1968, Ap\&SS, 2, 96

Fryxell, B., Olson, K., Ricker, P., et al. 2000, ApJS, 131, 273

Gal-Yam, A. 2012, Science, 337, 927

Gal-Yam, A., Mazzali, P., Ofek, E. O., et al. 2009, Nature, 462, 624

Gezari, S., Halpern, J. P., Grupe, D., et al. 2009, ApJ, 690, 1313

Glebbeek, E., Gaburov, E., de Mink, S. E., Pols, O. R., \& Portegies Zwart, S. F. 2009, A\&A, 497, 255

Heger, A., Langer, N., \& Woosley, S. E. 2000, ApJ, 528, 368

Heger, A., \& Woosley, S. E. 2002, ApJ, 567, 532

Heger, A., Woosley, S. E., \& Spruit, H. C. 2005, ApJ, 626, 350

Langer, N., Norman, C. A., de Koter, A., et al. 2007, A\&A, 475, L19

Lebovitz, N. R. 1967, ARA\&A, 5, 465

Leloudas, G., Chatzopoulos, E., Dilday, B., et al. 2012, A\&A, 541, A129

Maeder, A., \& Meynet, G. 2011, arXiv:1109.6171

Miller, A. A., Chornock, R., Perley, D. A., et al. 2009, ApJ, 690, 1303

Moriya, T., Tominaga, N., Blinnikov, S. I., Baklanov, P. V., \& Sorokina, E. I. 2011, MNRAS, 415, 199

Neill, J. D., Sullivan, M., Gal-Yam, A., et al. 2011, ApJ, 727, 15

Ofek, E. O., Rabinak, I., Neill, J. D., et al. 2010, ApJ, 724, 1396

Paxton, B., Bildsten, L., Dotter, A., et al. 2011, ApJS, 192, 3

Quataert, E., \& Shiode, J. 2012, MNRAS, 423, L92

Quimby, R. M., Kulkarni, S. R., Kasliwal, M. M., et al. 2011, Nature, 474, 487

Rakavy, G., \& Shaviv, G. 1968, Ap\&SS, 1, 429

Smith, N., Chornock, R., Li, W., et al. 2008, ApJ, 686, 467

Smith, N., Chornock, R., Silverman, J. M., Filippenko, A. V., \& Foley, R. J. 2010, ApJ, 709, 856

Smith, N., Li, W., Foley, R. J., et al. 2007, ApJ, 666, 1116

Smith, N., \& Owocki, S. P. 2006, ApJ, 645, L45

Spruit, H. C. 1999, A\&A, 349, 189

Spruit, H. C. 2002, A\&A, 381, 923

Timmes, F. X., \& Swesty, F. D. 2000, ApJS, 126, 501

Woosley, S. E., Blinnikov, S., \& Heger, A. 2007, Nature, 450, 390

Yoon, S.-C., Dierks, A., \& Langer, N. 2012, A\&A, 542, A113

Yoon, S.-C., Langer, N., \& Norman, C. 2006, A\&A, 460, 199

Yoshida, T., \& Umeda, H. 2011, MNRAS, 412, L78 